\title{
Virtual Prototyping of Large-Scale IoT Control Systems Using Domain-Specific Languages
}

\author{
Jacques Verriet ${ }^{1}$, Lennart Buit ${ }^{1}$, Richard Doornbos ${ }^{1}$, Bas Huijbrechts ${ }^{1}$, Kristina $\breve{S e v o}^{2}$, Jack Sleuters ${ }^{1}$ \\ and Mark Verberkt ${ }^{2}$ \\ ${ }^{1}$ ESI (TNO), Eindhoven, Netherlands \\ ${ }^{2}$ Signify, Eindhoven, Netherlands \\ \{jacques.verriet, richard.doornbos, bas.huijbrechts, jack.sleuters\}@tno.nl, \{kristina.sevo, mark.verberkt\}@signify.com
}

Keywords: Domain-specific languages, Model transformations, Virtual prototyping, System validation, Simulation, Model checking, Distributed control systems, IoT systems, Lighting systems

\begin{abstract}
IoT applications and other distributed control applications are characterized by the interaction of many hardware and software components. The inherent complexity of the distributed functionality introduces challenges on the detection and correction of issues related to functionality or performance, which are only possible to do after system prototypes or pilot installations have been built. Correcting these issues is typically very expensive, which could have been avoided by earlier detection. This paper makes four main contributions. (1) It presents a virtual prototyping approach to specify and analyze distributed control applications. The approach is based on a domain model, which can be configured for a specific application. It consists of eight domainspecific languages (DSLs), each describing one system aspect. (2) The DSLs provide each stakeholder in the application's lifecycle a natural and comprehensible way to describe his/her concerns in an unambiguous manner. (3) The paper shows how the DSLs are used to automatically detect common configuration errors and erroneous behavior. (4) The virtual prototyping approach is demonstrated using a lighting domain case study, in which the control system of an office floor is specified and analyzed.
\end{abstract}

\section{INTRODUCTION}

The cost of system development is dominated by verification and validation, which is typically done in the later development phases. Errors are often not detected until system prototypes or pilot installations reveal them and repairs have become costly. Correcting the issues found during these late phases is typically very expensive, whereas correction in the earlier phases of development has much lower cost (Haskins et al., 2004). The extra effort spent on early fault detection is much lower than the cost of fault detection and correction in the latest phases of development. Mellegård et al. (2016) and Broy et al. (2012) report similar findings.

Many errors are caused by ambiguity of requirement specifications. As requirements are typically specified in natural language, they can be interpreted in many ways. Similarly, design and architecture concepts are often described in an ambiguous manner (Theelen and Hooman, 2015; Völter, 2010).

This paper presents a virtual prototyping approach for IoT applications and similar distributed control applications. The approach allows unambiguous application specification as well as detection and correction of behavioral errors and performance issues in the early phases of development. The virtual prototyping approach is based on a domain model consisting of eight domain-specific languages (DSLs). These languages allow different aspects of an IoT application to be described in a non-ambiguous manner thereby avoiding misinterpretations. Each DSL describes one aspect of an IoT application and its environment. The behavior of an IoT application is described using DSLs describing (1) system topology, (2) system functionality, and (3) deployment of functionality onto the topology. Besides DSLs for system behavior, the domain model includes languages to describe the application's environment, i.e. the inputs it receives via its sensors.

As IoT applications may consist of thousands of components, their functionality cannot be tested easily. The systems are simply too large for a human to maintain overview of the state of the entire system. For this reason, the domain model includes a Requirement DSL in which one can specify the desired be- 
havioral properties. These properties are monitored as the prototype is running; violations of the specified properties are detected automatically. The scenarios leading to the property violation allow developers to diagnose the situation.

The domain model's DSLs form a configurable core, which describe an IoT system, its environment, and its requirements. This core can be extended using front-ends and back-ends for specific types of systems. We demonstrate this for the lighting domain. A real-life indoor lighting case study shows that the trade-off between lighting system performance and user comfort can be analyzed using a combination of (1) the domain model's DSLs, (2) usage profiles of people's activities in buildings, and (3) a lightingspecific interactive visualization.

\subsection{Contributions}

We make the following contributions to the state of the art. We present a virtual prototyping approach for distributed control applications, which can be configured for specific applications by defining specific event and structure types and coupling to custom environment models and visualizations. The domain model is based on a domain model consisting of eight DSLs, thereby providing each stakeholder in the application's lifecycle a natural and comprehensible way to describe his/her concerns in an unambiguous manner. The approach allows automatic detection of behavioral errors using executable models that are monitored for violation of specified requirements. The virtual prototyping approach is demonstrated using a real-life case study from the lighting domain.

\subsection{Outline}

The remainder of this paper is structured as follows. Section 2 provides an overview of related work. The domain model is introduced in Section 3. The validation capabilities of the domain approach are described in Section 4. The virtual prototyping approach is demonstrated using a real-life indoor lighting application case in Section 5. Conclusions are discussed in Section 6, future work in Section 7.

\section{RELATED WORK}

In this paper, we follow a similar approach as Hooman (2016), who proposes an approach to generate formal models from domain-specific languages. However, we use a modular domain model consisting multiple DSLs instead of a single DSL. Intro- ducing modularity allows a separation of concerns, better quality, and increased reuse (Rieger et al., 2018). Reuse of model elements is especially relevant for distributed control systems, as they typically have many components but only few component types. Like Hooman (2016), we combine our domain model with custom visualizations. Such graphical models have shown to improve communication between stakeholders (Broy et al., 2012) and speed up design (Beckers et al., 2007) in industry.

From our domain model, we automatically generate simulation models and model checking models. Simulations of large-scale IoT systems exist in literature, but they typically address the communication aspect, not the behavioral aspect of IoT systems. An example is the work of D'Angelo et al. (2017). To allow simulation of large-scale IoT systems, they apply a multi-level simulation. They combine a high-level simulator that operates on a wide scope and a coarsegrained level and low-level simulators that operate on a narrow scope and a fine-grained level. The latter are only used if fine-grained analysis is necessary.

DiaSuite (Bertran et al., 2014) does consider the behavioral aspect of IoT systems; it is a tool suite to develop, simulate and deploy sense-compute-control applications. It is based on Java-embedded DSLs, which describe the devices in a system including their attributes and interfaces. From this specification, abstract Java classes are generated. To allow simulation, programming skills are required: the desired behavior needs to be programmed.

Serral et al. (Serral et al., 2010) present PervML, a language to specify context-aware pervasive systems in a platform- and technology-independent manner. To specify pervasive systems, they distinguish two roles and multiple UML-based views. From a system specification, Java and OWL code is generated automatically.

France and Rumpe (France and Rumpe, 2007) observe that the specification and verification of system properties is important. This is another way in which our approach differs from that of Hooman (2016). As distributed control systems may contain thousands of components, it is practically impossible for a person to maintain overview of the system's behavior. For this reason, we include run-time monitors in the simulation and model checking models generated from our domain model. These monitors are used for automatic requirement checking.

There are many formalisms to describe these specifications. In the ComMA framework (Kurtev et al., 2017), specifications are based on MTL (Koymans, 1990), the real-time extension of LTL. Hendriks et al. (2016) uses MTL to check timed properties using ex- 
ecution traces. As it is difficult to specify and check LTL and MTL properties directly, there have been several initiatives to specify common monitoring patterns. Dwyer et al. (1998) defined a collection of temporal patterns; these were extended with timed patterns by Konrad and Cheng (2005) and Gruhn and Laue (2006). Meyers et al. (2013) describe a DSL-based method to specify system properties by combining temporal patterns. These are translated into LTL formulas that are checked using SPIN. Buit (2017) applies a similar technique for timed properties, which are translated into Uppaal timed automata. His work is used in this paper.

\section{DOMAIN MODEL}

We have developed a domain model consisting of eight DSLs to describe IoT applications and their environment. The languages and their usage relations are shown in Figure 1; this figure also shows the model-to-text transformations from the domain model's DSLs. We have used Xtext ${ }^{1}$ and Xtend ${ }^{2}$ for the development of the DSLs and transformations shown in Figure 1.

Figure 1 distinguishes three categories of languages: (1) Domain DSLs, (2) System DSLs, and (3) Validation DSLs. The Domain DSLs allow the generic domain model to be configured for a specific application; the Domain DSLs make the domain model a metamodel for distributed control applications. The System DSLs use the Domain DSLs to describe the structure and behavior of an IoT system. The Validation DSLs allow the validation of a specified system in its environment.

To explain the DSLs, we use a running example from the lighting domain. It involves a building with three rooms: two offices and a central lobby. Each room has one occupancy sensor and one light point.

The intended behavior in the offices can be specified in two rules: (1) When an office's occupancy sensor detects human presence and its light is off, then the office's light should switch on. (2) If the light in an office is on and no office occupancy is detected for a period of five minutes, then the office's light should switch off.

The behavior in the lobby is different: (1) If its light is off and occupancy is detected in an office or the lobby, then its light should switch on. (2) If the lobby's light is on and no occupancy is detected anywhere for a period of five minutes, then the lobby's light should switch off.

\footnotetext{
${ }^{1}$ http://www.eclipse.org/Xtext/

${ }^{2} \mathrm{http}: / /$ www.eclipse.org/xtend/
}

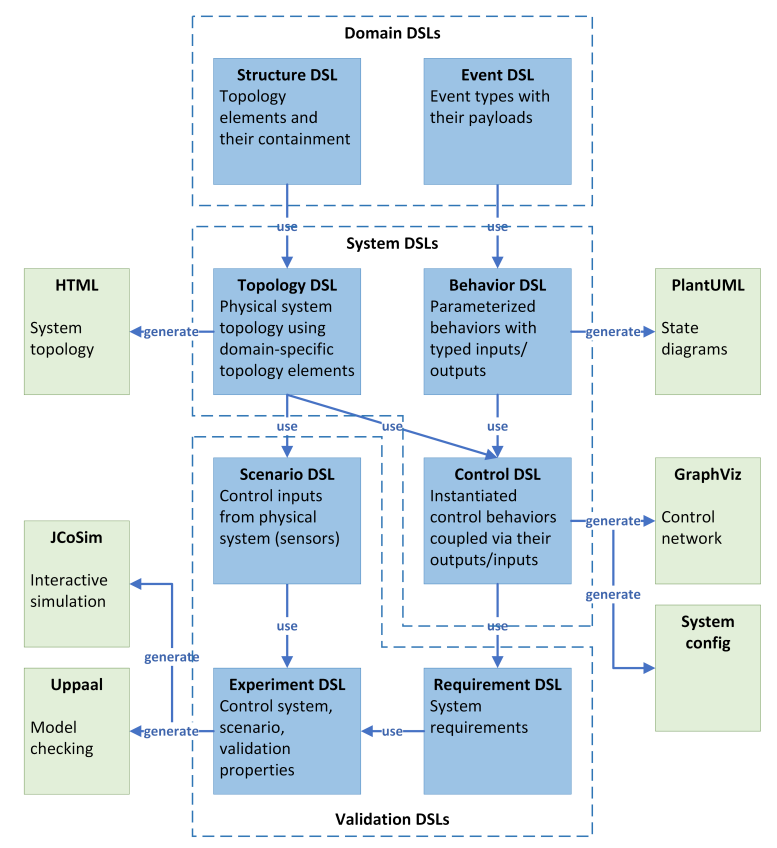

Figure 1: DSLs and model transformations.

\subsection{Domain DSLs}

As distributed control systems can be very extensive, it is beneficial to describe their structure hierarchically. Structure DSL allows the definition of such a (logical or physical) hierarchy. Instances of Structure DSL describe the types of structures in a distributed control system and the hierarchical containment of these structures.

For the reference example, we can use the logical structure of a building to describe a light control system. For this, we distinguish two types of structures: buildings and rooms. Rooms are assumed to be contained in buildings.

Event DSL describes the events that are communicated in the system. This includes the events that are sent from sensors and to actuators. Each event has a name and optional arguments.

For our running example, two types of events are distinguished: occupancy events are used to communicate occupancy and light level events are used to communicate a desired light level. Occupancy events do not have arguments; light level events have one, a light level represented by an integer value between 0 and 100 percent.

\subsection{System DSLs}

Topology DSL describes the (logical or physical) structure of a system in terms of the structure types defined in a Structure model. The class diagram of 


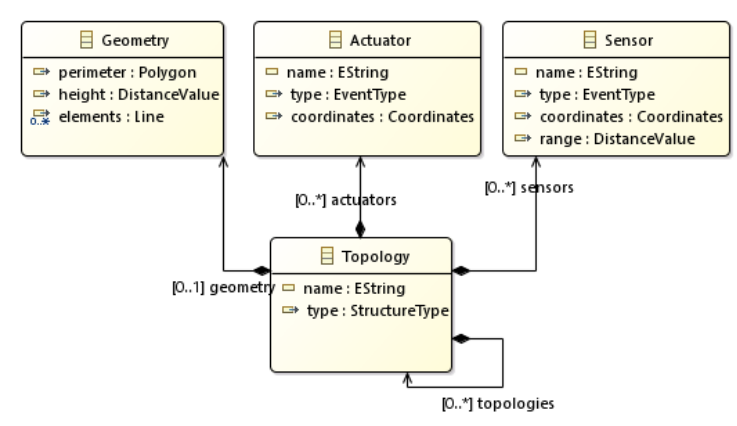

Figure 2: Topology DSL class diagram.

Topology DSL is shown in Figure 2. Topologies are built up hierarchically; each element of a topology has sensors and actuators, a physical geometry (used for visualization), and optional subordinate topologies.

Visualization is supported by a model transformation: for documentation purposes, Topology models can be translated into HTML pages with an SVG visualization of a system's hierarchical structure.

The running example's topology consists of four topological elements: a building and three rooms. The rooms are subordinate topologies of the building.

The specification of topology models for buildings need not be done manually; if information is stored in a Building Information Model (BIM) (Eastman et al., 2011), then a Topology model can be extracted from a BIM model automatically.

The desired behavior of an IoT system is realized by controllers that respond to sensory inputs. An IoT system may consist of thousands of controllers, but these typically have similar behaviors. This is exploited by Behavior DSL, which describes parameterized behaviors that can be instantiated for many different controllers. Behavior DSL is based on the Sense-Think-Act paradigm. The main classes of Behavior DSL are shown in Figure 3. System behaviors are described in terms of timed state machines. Transitions between states are triggered by input events or by timers and may be guarded by conditions. A transition involves issuing output events, updating local variables, and resetting timers.

A transformation to PlantUML ${ }^{3}$ is used to visualize behaviors; this transformation generates a graphical representation of the specified timed state machine from a (textual) Behavior model.

For the running example, we have specified the timed state machine shown in Figure 4. It involves two states and four transitions, two of which are selftransitions. The Vacant state is the behavior's initial state; this is the state in which the lights are off.

\footnotetext{
${ }^{3}$ http://www.plantuml.com/
}

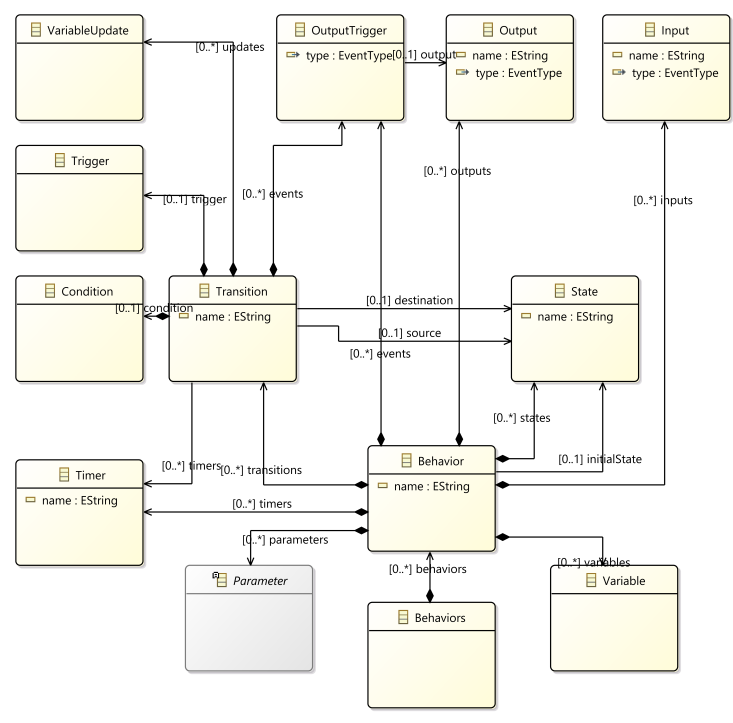

Figure 3: Behavior DSL class diagram.

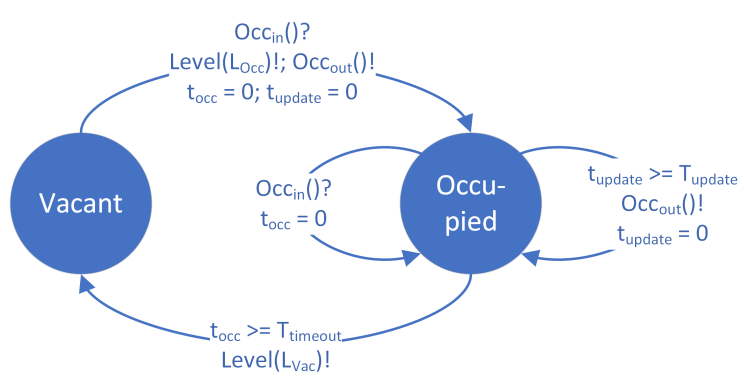

Figure 4: Behavior model.

When an occupancy event is received, a transition to the Occupied state is made. The transition also involves switching on the lights by issuing a light level event with argument $L_{\mathrm{Occ}}$. In addition, two timers are started, an occupancy timer and an update timer. In the Occupied state, an occupancy event is issued each time the update timer expires. This allows a controller to act as an occupancy sensor for other controllers. For example, the office controllers can provide occupancy input to the lobby controllers. In the Occupied state, the occupancy timer is reset each time an occupancy event is received. A timeout of the occupancy timer triggers a transition back to the Vacant state; this includes switching off the lights by issuing a light level event with argument $L_{\mathrm{Vac}}$.

Variation of a specific behavior is possible using a behavior's parameters, which can be set for each different instance. This is done in Control DSL, which instantiates behaviors for a given system. These instantiated behavior instances are called controllers. Control DSL also describes the mapping of behavior onto a system's topology. This is done by defining 
connections between sensors, controllers, and actuators. Sensors can be connected to controller inputs, actuators to controller outputs, and controller outputs to controller inputs. To allow analysis of the influence of network behavior, edges can be attributed with probability distributions for message loss and message latency. The class diagram of Control DSL is shown in Figure 5. To visualize the system structure, we have implemented a model transformation from Control DSL to GraphViz. ${ }^{4}$

We have also implemented code generators for lighting systems; these are model-to-text transformations from Control DSL. These transformations are less generic than the other transformations shown in Figure 1. This is because the specifics of the underlying technology are part of the model transformation. The generation of system configurations is outside the scope of this paper.

In our running example, we distinguish three controllers, one for each room. These controllers implement the behavior shown in Figure 4. The coupling of the sensors, controllers, and actuators of our reference example is shown in Figure 6. It shows the inputs and outputs of the room controllers. A room's sensor is connected to the room controller's occupancy input and the room controller's light level output to the room's actuator. In addition, the occupancy outputs of the office controllers are connected to the occupancy input of the lobby controller. This allows the lobby to respond to occupancy in the offices. In other words, the office controllers act as occupancy sensors of the lobby controller. The same can also be achieved by directly coupling the office sensors to the lobby controller, but this would involve many connections for large systems.

\subsection{Validation DSLs}

Scenario DSL provides a way to describe the behavior of the environment of a system; it describes sensor events at given moments in time. As each sensor event needs to be specified explicitly, Scenario DSL is suited for the specification of simple scenarios, e.g. test case scenarios. More complex scenarios can be specified using usage profiles, which should be calibrated using data from existing buildings. The profiles can be used to generate Scenario models or executable models that generate sensor triggers.

For our reference example, we do not include a Scenario model, because we validate the system using interactive simulation and exhaustive analysis using model checking.

\footnotetext{
${ }^{4} \mathrm{http} / / / w w w . g r a p h v i z . o r g /$
}

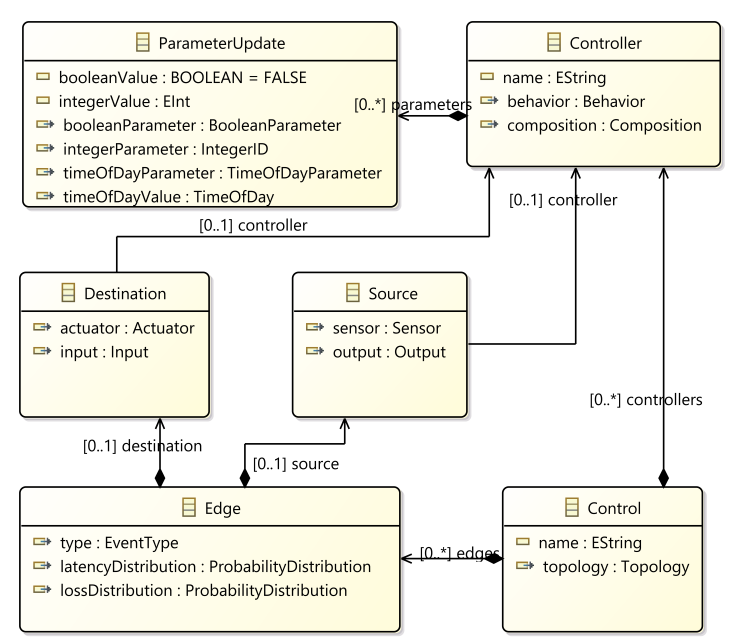

Figure 5: Control DSL class diagram.

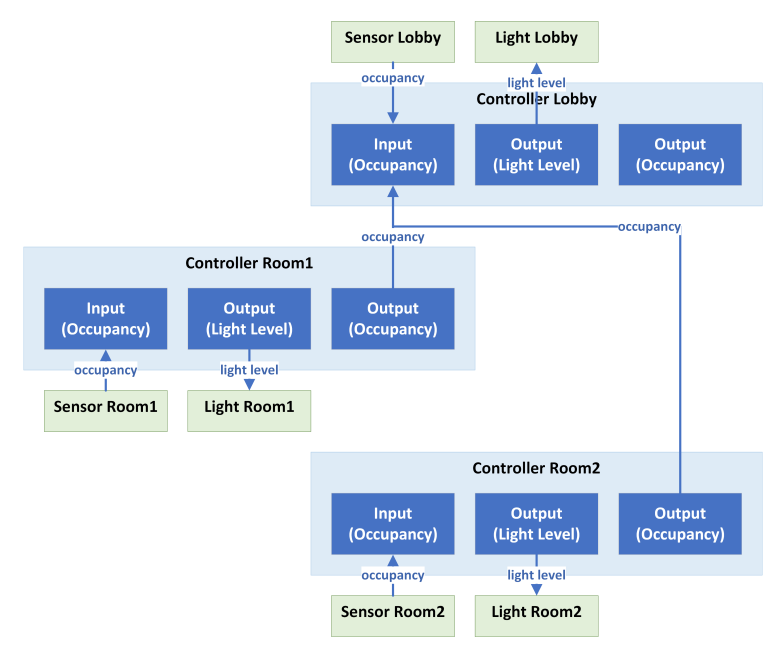

Figure 6: Control network.

Behavior DSL describes an IoT system's behavior in a procedural manner using states and transitions. In other words, it describes how the system should act. As specifying timed state machines is challenging, especially for large-scale systems and complex behaviors, we use Requirement DSL that describes what the system should do, i.e. in a declarative manner. For instance, Requirement DSL can specify what a system's response to a specific user scenario specified using Scenario DSL should be. It can, however, be used in a much wider setting. The separation of what and how also makes communication between technical and non-technical stakeholders easier, as acceptance requirements are not mixed up with technical details.

The main classes of Requirement DSL are shown in Figure 7. The language describes requirements in terms of propositions. Two types of propositions are 
distinguished: (1) event propositions hold for an infinitesimal time and (2) state propositions hold for longer time periods. Basic propositions can be combined in composite propositions, e.g. using Boolean operators and, or, and not. The circumstances under which these propositions should hold are described by a requirement's scope and pattern. Requirement DSL allows five scopes (Globally, After, Before, AfterUntil, Between) and seven patterns (Response, Precedence, Absence, Universality, Existence, Recurrence, Invariance), making a total of 35 pattern-scope combinations. Most patterns and scopes are based on those of Dwyer et al. (1998) and Konrad and Cheng (2005).

As IoT systems can become very extensive, it is very labor-intensive to specify requirements for all system components. Because such systems typically have many instances of the same behavior, Requirement DSL allows requirements to be specified for combinations of sensors, controllers, and actuators that adhere to a set of constraints. These combinations and the corresponding constraints are specified in a Requirement's header, which describes a pattern that is to be matched in a Control model. As such, Requirement DSL allows a highly efficient specification of system requirements: a requirement that should hold throughout an entire system needs to be specified only once.

For our reference example, we want to specify that when any office sensor detects occupancy, the lights in the lobby should switch/be on for at least five minutes. This requirement is shown in Figure 8. The requirement starts with a header specifying the sensors, controllers, and actuators that are to be considered. This example involves a sensor, two controllers, and an actuator; the sensor is connected to the first controller, the first controller to the second, and the second controller to the actuator. There are two such combinations: (1) Office1's sensor, Office1's controller, the Lobby's controller, and the Lobby's light point, and (2) Office2's sensor, Office2's controller, the Lobby's controller, and the Lobby's light point. For large-scale systems, there are typically many combinations that can be addressed using a single requirement.

In the requirement's body, two propositions are defined: the first proposition is an event proposition, which defines the occurrence of an occupancy event from the sensor; the second is a state proposition, which defines the actuator/light being fully on. The bottom statement is the actual requirement. This requirement uses the combination of the Globally scope and the Invariance pattern. This instance of the Invariance pattern specifies that each time the sensor is-

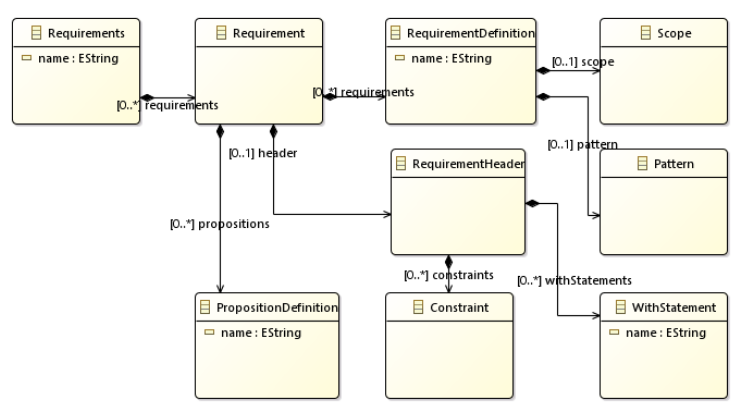

Figure 7: Requirement DSL class diagram.

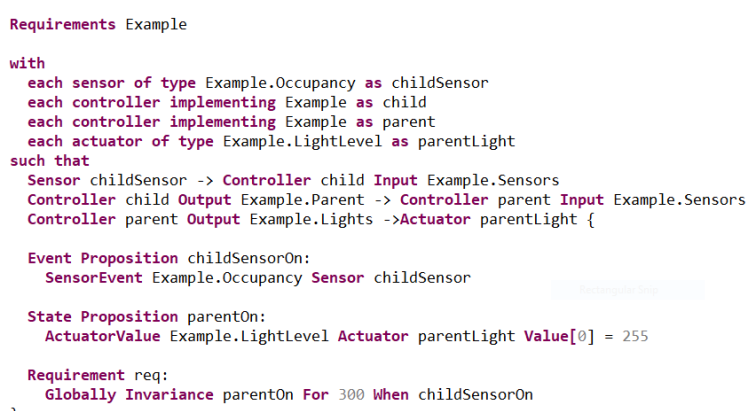

Figure 8: Requirement model.

sues an occupancy trigger, the actuator should be at $100 \%$ on for 300 seconds. The Globally scope indicates that this property should be satisfied under all circumstances.

Experiment DSL combines all information specified in the other DSLs; it combines instances of Control DSL, Scenario DSL, and Requirement DSL. The latter two are optional. As it includes the full system specification, Experiment DSL is the starting point for the transformation to analysis models. There are two transformations from Experiment DSL, one to a Java co-simulation framework and one to the model checker Uppaal (Larsen et al., 1995). These are explained in Section 4.

For the running example, an Experiment model is the combination of a Control and a Requirement model. It does not include a Scenario model.

\section{SYSTEM VALIDATION}

With our domain model, we support two types of system validation. Static system validation is discussed in Section 4.1. As not all system properties can be validated statically, we also support validation using executable models. Simulation is explained in Section 4.2, model checking in Section 4.3, and their combination in Section 4.4. 


\subsection{Static Validation}

Correctly specifying a large-scale IoT system is a huge challenge, as many concepts and settings have to be specified and it is difficult to keep overview. Because there are typically many similar structures and behaviors, people easily make specification errors, e.g. copy-paste errors. Our domain model provides support for avoiding errors and keeping overview, while the system is being specified.

For the DSLs introduced in Section 3, specific validation rules have been defined. Naming rules ensure uniqueness of model element names. This is relevant for nearly all languages, as the concepts introduced are used several times. For instance, event types are used by nearly all other DSLs.

Structure rules validate the structure of DSL instances. For a Behavior model's state machine, the reachability and leavability of all states is checked. For Topology models, it is checked whether the physical containment matches the logical containment. Control models are checked for cyclic dependencies between sensors, controllers, and actuators.

Usage rules validate usage and redundancy of defined concepts. For instance, it is checked whether the sensors and actuators in a Topology model and all inputs and outputs of a Control model are used. Moreover, it is checked whether actuators are connected to more than one controller, as this may cause nondeterministic behavior.

Type rules check type consistency. For instance, it is checked whether sources and destinations of edges in a controller network have the same event type. Moreover, it is checked whether controller parameters and event arguments are within the specified ranges.

\subsection{Simulation}

Simulation is used to validate the behavior and performance of IoT control systems. These systems may have thousands of sensors and actuators. To be able to run a simulation of an IoT system, one needs a platform that allows the simulation of many simultaneously executing and communicating simulators. To accomplish this, we have developed a lightweight Java co-simulation framework (JCoSim) providing timing and publish-subscribe services, similar to HLA's RTI (Dahman, 1997). We decided to develop our own services as the open-source versions of HLA did not offer the desired functionality at that time and the cost of the commercial versions were considered too high.

The transformation from Experiment DSL to JCoSim generates simulators from the components of

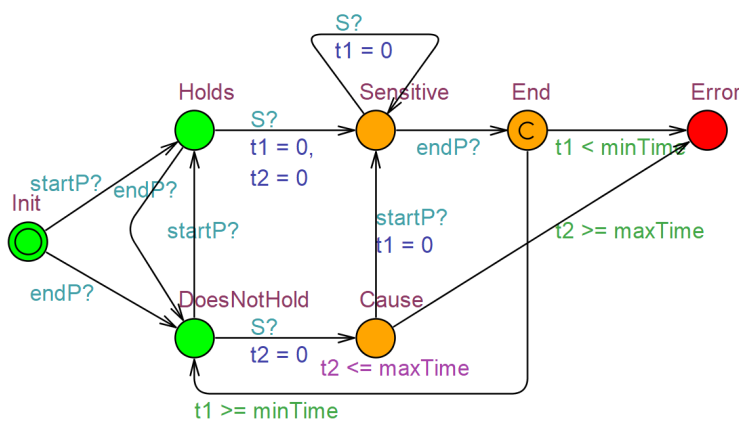

Figure 9: Timed automaton for Globally scope and Invariance pattern.

the specified IoT system. This involves separate simulators for sensors, controllers, and actuators. Moreover, edge simulators are generated to handle the communication in the control network; there is one simulator for each edge in a Control model. The Scenario model specifies the moments at which sensors trigger. This requires a scenario player simulator that triggers each sensor simulator to fire a sensor event at the right time.

Besides simulators for sensors, controllers, and actuators, the JCoSim model contains monitoring simulators, which are derived from a Requirement model. For the propositions and requirements specified in a Requirement model, proposition and requirement monitors are generated. Requirement DSL distinguishes basic and composite propositions; monitoring simulators are generated for both types. The monitoring simulators for the basic propositions monitor the controller simulators that are specified in a Control model; those for composite propositions observe other proposition simulators.

For each of the 35 pattern-scope combinations of Requirement DSL, a separate automaton has been defined and these are transformed into simulators in JCoSim. Figure 9 shows the automaton for the Globally scope and the Invariance pattern; the automata for the other scope-pattern combinations were defined by Buit (2017). The requirement simulators distinguish normal and forbidden state; the forbidden states represent requirement violations. During simulation, it is checked whether they enter a forbidden state. If they do, e.g. the Error state in the automaton in Figure 9, then the user is notified of the requirement violation.

For our running example, the transformation from Experiment DSL to JCoSim results in a model with 23 simulators: 3 sensor simulators, 3 controller simulators, 3 actuator simulators, 8 edge simulators, 4 proposition simulators, and 2 requirement simulators. 


\subsection{Model Checking}

Co-simulation provides a scalable system analysis method; it allows thousands of components to be simulated simultaneously. A drawback of simulation is the fact that it is limited to a single scenario, typically a good-weather scenario. As IoT systems may be very large and may have a lifespan of several decades, the analysis of good-weather behavior is not sufficient to find all errors that appear in a system's lifetime. We use model checking to analyze a system's behavior under all possible input scenarios. This has been realized by a transformation from Experiment DSL instances to Uppaal models (Larsen et al., 1995).

With respect to the generated automata, the transformation is very similar to the transformation to JCoSim. The only difference is that the generated Uppaal models do not have actuator automata; actuators are modeled using global variables. So whereas the JCoSim model for the reference example involves 23 simulators, the corresponding Uppaal model has only 20 automata.

There are some conceptual differences between the timed state machines specified in Behavior DSL and Uppaal's timed automata. The main differences are: (1) Uppaal does not allow multiple synchronizations per transition and (2) Uppaal does not allow synchronizations with data. The former has been addressed by introducing sequences of synchronizations between committed states; these committed states make sequences of transitions atomic. We addressed the latter by communication using global variables.

The Uppaal model contains the same proposition and requirement automata as the JCoSim simulation model. JCoSim is restricted to monitoring whether forbidden states are entered. Besides entering of forbidden states, the Uppaal models allows other types of analysis. An overview of the properties that can be checked is given by Buit (2017).

For our running example, an Uppaal model with 20 automata and 2 queries is generated, one query per requirement automaton. Uppaal's exhaustive analysis shows that both queries are satisfied: i.e. when a sensor in an office detects occupancy, then the lights in the lobby are/switch on for at least five minutes.

\subsection{Simulation and Model Checking}

Model checking provides an exhaustive system analysis; this means that it finds requirement violations even under very unlikely circumstances. Unfortunately, model checking is not sufficiently scalable to analyze the (possibly) huge state space of an IoT system. This does not mean that model checking does not provide value for the analysis of complex systems. To manage complexity, our approach breaks down the system into manageable subsystems using the the Control model as a basis. Instead of analyzing all controllers and all requirements simultaneously, one can, similar to the approach of Doornbos et al. (2015), iteratively analyze all combinations of one controller and one requirement. The state space of these combinations is typically small enough to allow exhaustive analysis.

A second way in which model checking adds value is by combining the strengths of simulation and model checking. Simulation is sufficiently scalable to simulate large-scale systems, but large-scale simulations provide limited diagnostic capabilities. If simulation detects a requirement violation in a system, then model checking can be used for diagnosis. Model checking should zoom in on the controllers causing the violation. As a requirement violation is known to exist, model checking's exhaustive analysis will find it and provide a minimal trace leading to the violation. This trace is translated into a Scenario model that can be used to identify the underlying root cause.

\section{CASE STUDY}

The domain model introduced in Section 3 has been applied for intelligent lighting systems. Such systems typically consist of devices connected via an IP communication network. These devices need not be restricted to the lighting domain as lighting systems are more and more integrated with other building management systems, such as HVAC, blinds, and elevators. The input devices include a variety of sensors (e.g. occupancy and light level sensors), buttons for scene selection or dimming, but also mobile devices for personalized control. A lighting system's output devices are light points, units consisting of one or more LED units with all necessary parts and wiring.

An existing building has been used as the basis for a case study of our domain model: Witte Dame ${ }^{5}$ is a renovated factory in the center of Eindhoven. In the context of the OpenAIS project, ${ }^{6}$ the traditional lighting system on the building's fifth floor has been replaced by a new intelligent lighting system. This system was modeled and simulated using our domain model. The light system covers 367 light points with over 1,300 behavioral functions. The floor plan is shown in Figure 10.

\footnotetext{
${ }^{5}$ http://www.dewittedame.nl/

${ }^{6} \mathrm{http}: / /$ www.openais.eu/
} 


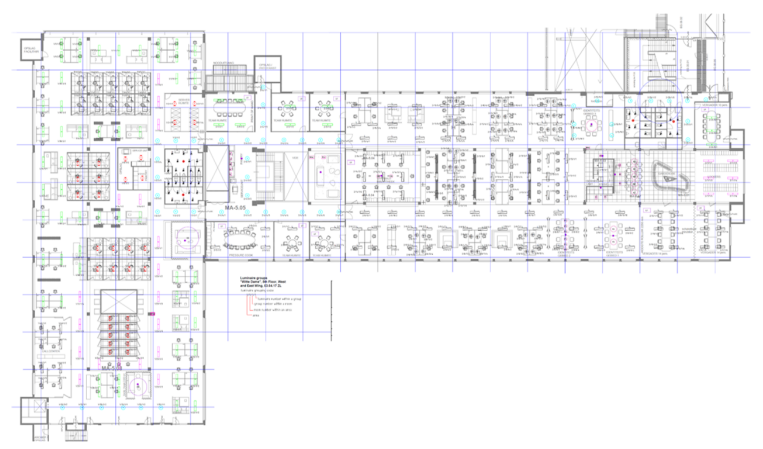

Figure 10: Floor plan of Witte Dame's fifth floor.

The first step in the case study involved the validation of the intended Behaviors. This was done by deploying a Behavior on a relevant part of Witte Dame and validating whether the deployed Behavior was as expected. For this, we used the simulation and model checking as explained in Section 4. To allow discussion with stakeholders, we developed visualization tooling that allows interactive simulation. A user can trigger sensors in the interactive visualize and observe the resulting system behavior.

The combination of Requirement DSL and the interactive visualization has shown its great value. While simulating a lighting system, the status of the sensors and light points is visualized. For instance, the status of sensors and buttons is shown and the light level of light points. In addition, the status of the requirements is shown using a transparent overlay over the corresponding devices. A requirement's overlay starts green and turns red when the requirement is violated.

The second step involved the validation of the system. Manually creating DSL instances for hundreds of light points would be a lot of effort and would be extremely error prone. Hence we developed a pipeline of model transformations (Rieger et al., 2018). Dedicated tooling has been developed to generate Control models. The corresponding work flow is visualized in Figure 11. There are two inputs: (1) a Microsoft Visio file describing the geometry of the floor plan including the location of the sensors and light points, and (2) a Microsoft Excel document describing the system behavior and their deployment onto the devices in the system. From this and manually created Structure, Event, and Behavior models, two models are generated: (1) a Topology model and (2) a Control model. From these generated models, Java simulation models are generated as explained in Section 4.2.

To thoroughly test a lighting system using simulation, elaborate scenarios need to be specified. Because Scenario DSL is not suited to manually specify long scenarios and manually triggering a gener-

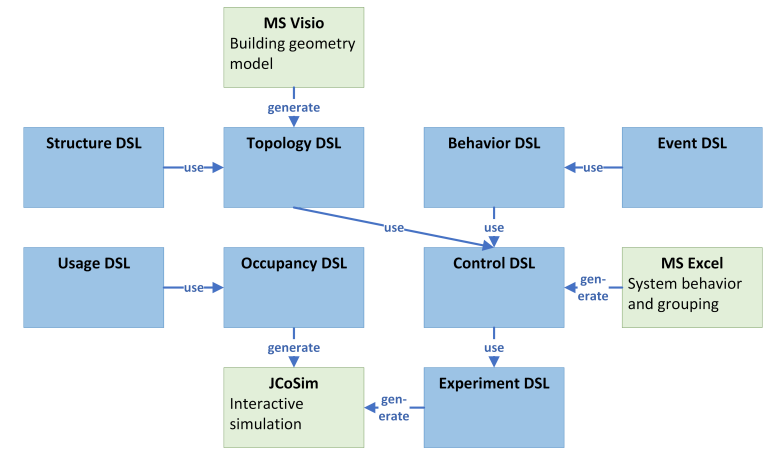

Figure 11: Generation of co-simulation models.

ated lighting system is both tedious and inefficient, we have developed Usage DSL and Occupancy DSL specifically for human behavior in buildings. These languages allow people's activities and their mapping onto locations in a building to be specified. The simulation of people's movement and activities in the building generate sensor triggers that automatically trigger the generated lighting simulation.

A screenshot of the interactive simulation is shown in Figure 12. It shows Witte Dame's floor plan and the status of the light points in the system. Apart from lighting behavior, the simulation also considers the system's performance with respect to energy usage, an important KPI for lighting systems (Baumgartner et al., 2012). The virtual prototype allows an assessment of the energy usage for different behaviors and behavior settings. For instance, one can analyze the influence of occupancy timeout periods on a lighting system's energy usage.

The co-simulation has proven to be very useful in the design of the large-scale virtual prototype. During the verification of the control behavior of Witte Dame more than ten errors have been identified and corrected. These included (1) incorrect or missing control group allocations of light points, (2) incorrect sensor linking, and (3) missing or incorrect linking of controllers. Although these errors could have been found by manual inspection, co-simulation greatly accelerated the errors' detection and correction.

Furthermore, the validation of the behavior with lighting experts has led to renewed discussions on customer-desired behavior leading to several adaptations to the initial control design for the Witte Dame prototype including improved controller linking and better behavior specification. These results clearly illustrate the power of the co-simulation environment. 


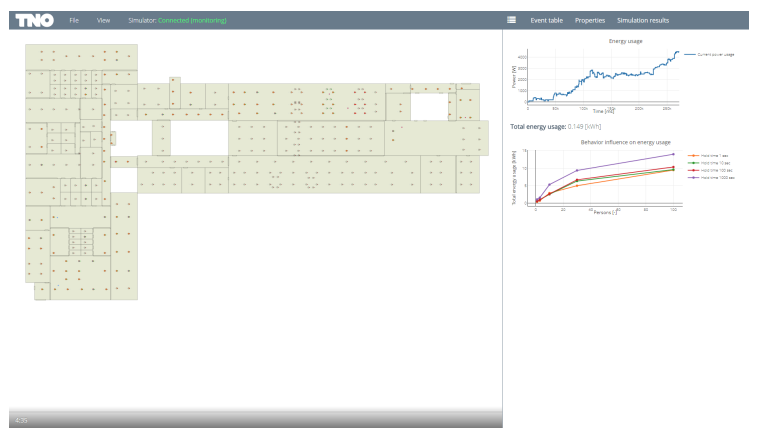

Figure 12: Simulation of Witte Dame case study.

\section{CONCLUSION}

In this paper, we have presented a domain model for distributed control systems, which comprises eight DSLs that each capture one system aspect. For instance, it includes two languages that allow the generic domain model to be adapted for a certain domain. Moreover, the system's structure is separated from its behavior, allowing reuse of behavior in multiple locations.

The domain model is the basis for a virtual prototyping application, which supports both simulation and model checking. For both kinds of analysis, we have separated what the system should do from how it does this. This provides powerful analysis support for large-scale distributed control systems: Requirement DSL is the basis for the generation of monitoring automata that are included in the generated simulation and model checking models. These automata observe the system and notify the user of requirement violations. The combination of simulation and model checking is of especially great value: (1) simulation allows large systems and many requirements to be analyzed simultaneously, and (2) model checking allows diagnosis of an identified requirement violation by zooming in on the violating subsystem.

Our co-simulation framework allows scalable simulation of thousands of sensors, controllers, and actuators. In the lighting domain, we have shown that the combination of our generic co-simulation with domain-specific front-ends and back-ends is very valuable: (1) usage and occupancy front-ends were used to generate realistic building occupancy patterns, and (2) a visualization back-end was used to visualize system behavior and allow users to interact with a running simulation. The interactive visualization allows early feedback of system behavior, possibly already during a system's sales phase.

\section{FUTURE WORK}

The domain model described in Section 3 is focused on the interaction between distributed sensors, controllers, and actuators. Implicitly, it is assumed that this interaction does not involve complex data being shared. For some distributed control systems, data plays a prominent role. For instance, in the logistic domain, one needs to keep track of orders and resources. To include such concepts, our domain model needs to be extended by a Data DSL, which is used to define data structures, which can be used by the other DSLs. This would allow analysis of logistic control systems such as the ones studied by Verriet et al. (2012).

The system validation described in Section 4 proposes simulation and model checking to detect and correct system errors. Not all errors can be addressed using these techniques: e.g. configuration errors can be found, but hardware failures cannot. To allow more types of errors to be found, a root cause analysis approach is proposed. This approach feeds our virtual prototype with actual sensor data and compares the response of the virtual prototype's actuators to the actual actuator responses. In case of differences, a reasoning framework is to be used to identify the most probable root cause.

The domain model described in this paper allows the specification of distributed control systems. The desired system behavior can be realized in many ways. For instance, one may decide to have redundant controllers to improve system reliability. The necessary communication of the redundant controllers can be specified in terms of Behavior models. This mixes functional and non-functional system aspects. A challenging open issue is the separation of these aspects. Specifically, we would like to specify system behavior in a deployment-agnostic manner and describe deployment, and the corresponding communication, in a separate Deployment DSL. This would allow efficient analysis of different deployment strategies, as the behavior needs to be specified only once.

\section{ACKNOWLEDGEMENTS}

The research is carried out as part of the Prisma program and H2020 OpenAIS project under the responsibility of Embedded Systems Innovation (ESI) with Philips Lighting as the carrying industrial partner. The Prisma program is supported by the Netherlands Ministry of Economic Affairs, the OpenAIS project is co-funded by the Horizon 2020 Framework Programme of the European Union under grant agree- 
ment number 644332 and the Netherlands Organisation for Applied Scientific Research TNO.

\section{REFERENCES}

Baumgartner, T., Wunderlich, F., Jaunich, A., Sato, T., Bundy, G., Grießmann, N., and Hanebrink, J. (2012). Lighting the way: Perspectives on the global lighting market. Technical report, McKinsey \& Company, Inc.

Beckers, J. M. J., Muller, G. J., Heemels, W. P. M. H., and Bukkems, B. H. M. (2007). Effective industrial modeling for high-tech systems: The example of happy flow. In 17th INCOSE International Symposium, pages 1758-1769, San Diego, CA. INCOSE.

Bertran, B., Bruneau, J., Cassou, D., Loriant, N., Balland, E., and Consel, C. (2014). Diasuite: A tool suite to develop sense/compute/control applications. Sci. Comput. Program., 79:39-51.

Broy, M., Kirstan, S., Krcmar, H., Schätz, B., and Zimmermann, J. (2012). What is the benefit of a model-based design of embedded software systems in the car industry? In Emerging Technologies for the Evolution and Maintenance of Software Models, pages 343-369. IGI Global, Hershey, PA.

Buit, L. J. (2017). Developing an easy-to-use query language for verification of lighting systems. Master's thesis, University of Twente, Enschede.

Dahman, J. S. (1997). High level architecture for simulation. In 1st International Workshop on Distributed Interactive Simulation and Real Time Applications, pages 9-14, Eilat. IEEE.

D'Angelo, G., Ferretti, S., and Ghini, V. (2017). Multi-level simulation of internet of things on smart territories. Simul. Model. Pract. Theory, 73:3-21.

Doornbos, R., Verriet, J., and Verberkt, M. (2015). Robustness analysis for indoor lighting systems: An application of model checking in large-scale distributed control systems. In 10th International Conference on Systems, pages 46-51, Barcelona. IARIA.

Dwyer, M. B., Avrunin, G. S., and Corbett, J. C. (1998). Property specification patterns for finite-state verification. In 2nd Workshop on Formal Methods in Software Practice, pages 7-15, Clearwater Beach, FL. ACM.

Eastman, C., Teicholz, P., Sacks, R., and Liston, K. (2011). BIM Handbook: A Guide to Building Information Modeling for Owners, Managers, Designers, Engineers and Contractors. Wiley, Hoboken, NJ.

France, R. and Rumpe, B. (2007). Model-driven development of complex software: A research roadmap. In 2007 Workshop on the Future of Software Engineering, pages 37-54, Minneapolis, MN. IEEE.

Gruhn, V. and Laue, R. (2006). Patterns for timed property specifications. Electron. Notes Theor. Comput. Sci., 153:117-133.

Haskins, B., Stecklein, J., Dick, B., Moroney, G., Lovell, R., and Dabney, J. B. (2004). Error cost escalation through the project life cycle. In 14th INCOSE International Symposium, pages 1723-1737, Toulouse. INCOSE.
Hendriks, M., Geilen, M., Behrouzian, A. R. B., Basten, T., Alizadeh, H., and Goswami, D. (2016). Checking metric temporal logic with trace. In 16th International Conference on Application of Concurrency to System Design, pages 19-24, Torun. IEEE.

Hooman, J. (2016). Industrial application of formal models generated from domain specific languages. In Theory and Practice of Formal Methods, volume 9660 of Lecture Notes in Computer Science, pages 277-293. Springer, Berlin.

Konrad, S. and Cheng, B. H. (2005). Real-time specification patterns. In 27th International Conference on Software Engineering, pages 372-381, St. Louis, MO. ACM.

Koymans, R. (1990). Specifying real-time properties with metric temporal logic. Real-Time Syst., 2:255-299.

Kurtev, I., Hooman, J., and Schuts, M. (2017). Runtime monitoring based on interface specifications. In ModelEd, TestEd, TrustEd, volume 10050 of Lecture Notes in Computer Science, pages 335-356. Springer, Berlin.

Larsen, K. G., Pettersson, P., and Yi, W. (1995). Modelchecking for real-time systems. In International Symposium on Fundamentals of Computation Theory, volume 965 of Lecture Notes in Computer Science, page 62-88. Springer, Berlin.

Mellegård, N., Ferwerda, A., Lind, K., Heldal, R., and Chaudron, M. R. V. (2016). Impact of introducing domain-specific modelling in software maintenance: An industrial case study. IEEE Trans. Softw. Eng., 42:245-260.

Meyers, B., Wimmer, M., van Vangheluwe, H., and Denil, J. (2013). Towards domain-specific property languages: The promobox approach. In 2013 Workshop on Domain-specific Modeling, pages 39-44, Indianapolis, IN. ACM.

Rieger, C., Westerkamp, M., and Kuchen, H. (2018). Challenges and opportunities of modularizing textual domain-specific languages. In 6th International Conference on Model-Driven Engineering and Software Development, pages 387-395, Funchal. SciTePress.

Serral, E., Valderas, P., and Pelechano, V. (2010). Towards the model driven development of context-aware pervasive systems. Pervasive Mob. Comput., 6:254-280.

Theelen, B. and Hooman, J. (2015). Uniting academic achievements on performance analysis with industrial needs. In Quantitative Evaluation of Systems, volume 9259 of Lecture Notes in Computer Science, pages 3 18. Springer, Berlin.

Verriet, J., Liang, H. L., Hamberg, R., and van Wijngaarden, B. (2012). Model-driven development of logistic systems using domain-specific tooling. In Complex Systems Design \& Management 2012, pages 165-176, Paris. Springer.

Völter, M. (2010). Architecture as language. IEEE Softw., 27:56-64. 